\title{
Construction and analysis of a library of miRNA in gold-coloured mutant leaves of Ginkgo biloba L.
}

\author{
Weixing Li, Zhichong He, Shunbo Yang, Yunling Ye, Huiru Jiang, Li Wang*
}

College of Horticulture and Plant Protection

Yangzhou University, Yangzhou 225009, China

\begin{abstract}
To gain insights into the regulatory networks of miRNAs related to golden colour formation in Ginkgo biloba leaves, we constructed an sRNA library of golden-green striped mutant leaves. A total of 213 known miRNAs comprising 54 miRNA families were obtained, and 214 novel miRNAs were identified in the mutant leaves. We further constructed a normal green leaf sRNA library as a control and compared the expression of miRNAs between mutant and normal leaves. We found 42 known and 54 novel differential expression candidate miRNAs; 39 were up-regulated and 57 down-regulated in mutants compared to normal leaves. Our transcriptome analysis and annotation of the predicted targets indicated that the potential roles of miRNAs in G. biloba leaves included involvement in the 'Glutathione metabolism', 'Plant circadian rhythm', and 'Phenylalanine metabolism' categories. miRNAs and their targets were further validated by qRT-PCR. The expression of miR159a and miR159c, in particular, was significantly higher in mutant leaves than in normal leaves, while their potential target gene $C L T 3$, which is associated with chloroplast development, displayed the opposite expression pattern. In addition, the expression of miR396g-3p and miR396h was also significantly higher in mutant leaves than in normal leaves, while the target genes $A B P 1$ (auxin-related gene) and PPR32 (chloroplast RNA editing protein), respectively, showed the opposite expression pattern. Combined with the transcriptome analysis, these data suggest that miR159, miR396, and their targets may participate in chloroplast development and hormone metabolism to regulate colour formation in G. biloba leaves.
\end{abstract}

Key words: chloroplast development, Ginkgo biloba, leaf coloration, leaf yellowing, microRNAs

\begin{abstract}
Abbreviations:
ABP - auxin-binding protein, ARC - accumulation and replication of chloroplasts, ARF - auxin response factor, CLT - CRT-like transporter, GBG - Ginkgo biloba green leaves, GBY - Ginkgo biloba yellow leaves, GLK - Golden2-Like, PI - phytochrome interacting factors, PPR - pentatricopeptide repeat, SPL - Squamosa promoter binding protein-like
\end{abstract}

\section{INTRODUCTION}

Leaf colour is an important appearance characteristic in ornamental plants. Many plant species change their leaf colours in different developmental stages or environmental conditions. Generally, the colour of leaves is directly determined by changes in the contents, especially the proportions of chlorophyll, carotenoids, and anthocyanins. The yellowing of leaves is mostly due to a decrease in the chlorophyll content in the leaves, or an increase in yellow pigments such as carotenoids, which leads to an increased proportion of yellow pigments (Archetti,

\footnotetext{
*Corresponding author. 
2000; Lee et al., 2003). In the bleached leaves of Arabidopsis, the leaf colour phenotype is due to chloroplast abnormalities (Garton et al., 2007). Moreover, some leaf colour mutants of Zea mays, Oryza sativa and A. thaliana have also been proved to be caused by chlorophyll deficiency (Li et al., 2012; Lonosky et al., 2004; Maekawa et al., 2015). The inhibition of chloroplast DNA replication results in fewer chloroplasts and induces the yellow phenotype of leaves. Chlorophyll synthesis involves more than 15 kinds of enzymes and 20 genes (Adhikari et al., 2011; Nagata et al., 2005). Some important functional genes, such as HEMAI, $C H L H$, and $C A O$, become less active during the process of leaf colour transformation (Mochizuki et al., 2001; Papenbrock et al., 2000; Wu et al., 2007). Additionally, $\mathrm{NYCl}$ and $\mathrm{NOL}$ play key roles in the process of chlorophyll degradation (Horie et al., 2009; Sato et al., 2007). Some transcription factors also play important roles in the biosynthesis and metabolism of pigments. For example, the GLK, $\mathrm{ARC}$, and PIF transcription factor families regulate leaf chlorophyll biosynthesis directly or indirectly (Maple and Mãller, 2007; Moon et al., 2008; Pan et al., 2013). Overall, chloroplast development and various transcription factors all participate in leaf coloration.

miRNAs have been demonstrated to be involved in the synthesis and metabolism of plant pigments. For example, anthocyanins accumulate in the junctions of rosettes and stems of A. thaliana, and they are mainly regulated by the miR 156 target gene SPL9. miR156 can significantly inhibit the expression of SPL9, and activate the expression of key synthetic anthocyanin genes to increase anthocyanin synthesis (Eriksson et al., 2004). miR858 was predicted to target MYB12 in A. thaliana. In myb12-deletion mutant plants, the synthesis of flavonoids was reduced, while myb12overexpression plants contained more flavonoids, suggesting that miR858 might regulate flavonoid metabolism (Guan et al., 2014). In addition, some miRNAs may be involved in the regulation of carotenoid synthesis and accumulation. However, most studies have focused on model plants, such as A. thaliana, whereas woody plant studies are still lacking.

Ginkgo biloba L. is a landscape tree famous for its golden leaves in autumn. Previously, we found a novel golden-green striped leaf mutant and investigated the mutant leaves in cytological, physiological, and transcriptomic analyses (Li et al., 2018). Cytological and physiological experiments showed that the mutant had an abnormal ultrastructure in the chloroplast together with a lower chlorophyll content. We also identified various chlorophyll metabolism-related genes, which might be involved in leaf coloration in the mutant. However, any miRNA-mediated coloration remains unknown. Therefore, in this study, we constructed libraries of small RNAs of GBG and GBY. Through differential expression analysis, we identified differentially expressed miRNAs associated with chloroplast development and hormone metabolism that might be involved in colour formation through the regulation of chloroplast development.

\section{MATERIAL AND METHODS}

\section{Plant material}

In this study, we used a $G$. biloba mutant tree with golden-green striped leaves growing under natural conditions at Yangzhou University, Jiangsu province, China $\left(32^{\circ} 39^{\prime} \mathrm{N}, 119^{\circ} 43^{\prime} \mathrm{E}\right)$. Healthy leaves, including normal green leaves and yellowgreen striped leaves, were collected from the same short shoot separately from May to July in 2015 and 2016 (Fig. 1). The two types of sampled leaves were rapidly cut into pieces and mixed separately, then all the samples were immediately frozen in liquid nitrogen and stored at $-80^{\circ} \mathrm{C}$ until use.

\section{Small RNA sequencing}

Two libraries of small RNAs (sRNAs), one each from normal and mutant leaves, were constructed and sequenced by Novogene Biotechnology Corporation (Beijing, China) using an Illumina Hiseq $^{\text {TM }} 2500$ sequencing platform. Briefly, total
(A)

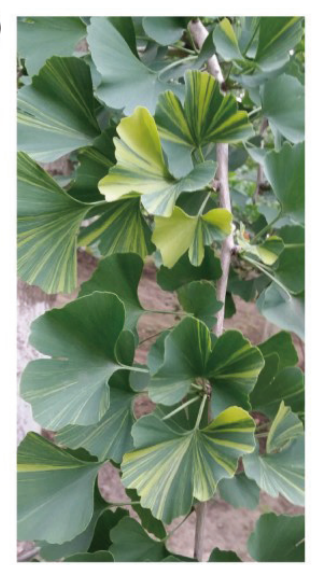

(B)

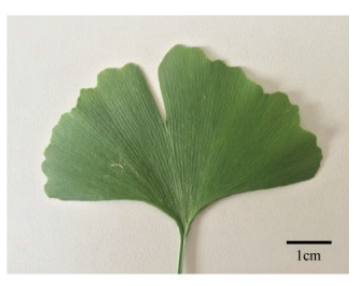

(C)

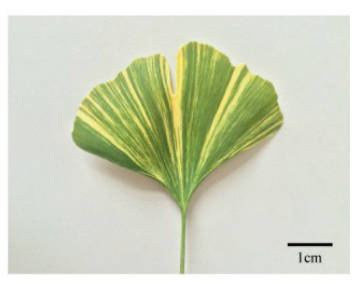

Figure 1. Normal green leaves and mutant leaves of Ginkgo biloba in May: (A) normal green leaves and mutant leaves on the same short shoot of G. biloba; (B) a normal green leaf; (C) a mutant leaf. Scale bar: $1 \mathrm{~cm}$ 
RNA was extracted from G. biloba green (GBG) and yellow leaves (GBY) with a TaKaRa Mini BEST Plant RNA Extraction Kit (TaKaRa, Dalian, China) according to the manufacturer's protocol. After checking the quality and yield, $10 \mu \mathrm{g}$ of high quality RNA from each sample was used to construct the sRNA library, and the two libraries were generated using a NEBNext ${ }^{\circledR}$ Multiplex Small RNA Library Prep Set for Illumina ${ }^{\circledR}$ (NEB, Ipswich, MA, USA) according to the manufacturer's protocol. Finally, single-end reads (read length: $50 \mathrm{bp}$ ) were obtained and then used for subsequent analysis.

\section{Identification of known and novel miRNAs}

Sequenced reads (raw data) were trimmed by removing low-quality and contaminated reads. The clean reads were mapped to the reference sequences by Bowtie. Small RNA tags without mismatch were analyzed for their expression and distribution on the reference. Then, perfectly matched sRNA sequences were used to query against RepeatMasker, the Rfam database, or species-specific databases, and then the sRNAs originating from repeat sequences, protein-coding genes, rRNAs, tRNAs, snRNAs and snoRNAs were excluded. The remaining unique sRNAs were aligned against miRBase 20.0 to seek for known miRNAs (without mismatch). Finally, the remaining unannotated sequences were used to predict novel miRNAs by miREvo and mirdeep2 software, through exploring the secondary structure, the Dicer cleavage site and the minimum free energy.

\section{miRNA expression analysis and miRNA target prediction}

miRNA expression levels were estimated by TPM (transcripts per million) criteria: normalized expression $=$ mapped read count $/$ total mapped reads $\times 1000000$. Using the DESeq $\mathrm{R}$ package, differential expression of the two leaf samples was analyzed. Expression differences greater than twofold (fold change $\log _{2}>1$ or $<-1$ and $p<0.01$ ) between the two libraries were set as the default thresholds to identify differentially expressed miRNAs.

The putative targets of known and novel miRNAs were identified by the web-based psRNATarget program (http://plantgrn.noble.org/psRNATarget/) and psRobot_tar in psRobot. The psRobot software was used to evaluate all the predicted target genes. Genes with a score $<3$ were considered as miRNA targets. The miRNA sequence data reported in this paper have been deposited in the Gene Expression
Omnibus (GEO) database under the accession number GSE122410.

\section{qRT-PCR validation of miRNAs and targets}

For qRT-PCR of miRNAs, RT synthesis (about $1 \mu 1$ RNA solution) was performed using a miRcute miRNA First-Strand cDNA Synthesis kit (Tiangen, Beijing, China) according to the manufacturer's instructions. The forward primers for 18 miRNAs were also designed using Primer Premier 5.0 (Tab. 1), and the reverse primers were provided by a miRcute Plus miRNA qPCR Kit (SYBR Green) (Tiangen, Beijing, China). The PCR reactions were performed as follows: $95^{\circ} \mathrm{C}$ for $15 \mathrm{~min}$., followed by 40 cycles of $94^{\circ} \mathrm{C}$ for $20 \mathrm{~s}, 60^{\circ} \mathrm{C}$ for $34 \mathrm{~s}$.

For qRT-PCR of miRNA targets, total RNAs of the two types of leaves were extracted as described above and the cDNAs were synthesized using a PrimeScript ${ }^{\mathrm{TM}}$ Reverse Transcriptase Reagent Kit with gDNA Eraser (Perfect Real Time; TaKaRa). Four pairs of primers for miRNA targets were designed using Primer Premier 5.0 (Tab. 2). The qRT-PCR reactions used no more than $100 \mathrm{ng}$ cDNA, $12.5 \mu$ l SYBR Premix Dimer Eraser (2×), and $0.3 \mu \mathrm{M}$ of each primer. The qRT-PCR program was as follows: denaturation at $95^{\circ} \mathrm{C}$ for $30 \mathrm{~s}$, followed by 40 cycles of $95^{\circ} \mathrm{C}$ for $5 \mathrm{~s}, 55^{\circ} \mathrm{C}$ for $30 \mathrm{~s}$, and $72^{\circ} \mathrm{C}$ for $30 \mathrm{~s}$. All reactions were conducted on a CFX96 Detection System (Bio-Rad, USA). Three biological and technical replicates were performed in the qRT-PCR experiment. The relative expression levels of miRNAs and their targets were calculated and normalized to the internal control U6 and GAPDH, respectively, using the $2^{-\Delta \Delta C t}$ method (Livak and Schmittgen, 2001). Ct represents the threshold cycle. Standard errors of the mean among the replicates were calculated. Significance analyses comparing GBG and GBY were also conducted.

\section{RESULTS}

\section{Sequencing and analysis of small RNAs in mutant leaves of G. biloba}

To identify miRNAs involved in the mutant leaves of $G$. biloba, a library of small RNAs from the mutant leaves was constructed and sequenced on the Illumina $\mathrm{HiSeq}^{\mathrm{TM}} 2500$ platform. For subsequent analysis, sequences were aligned to the reference G. biloba genome sequence, and those that matched the genome sequence perfectly were subjected to further computational analysis. A total of 28,827,507 raw reads were obtained. After removing sequences $<18 \mathrm{nt}$, poly-A sequences, 
Table 1. Forward primers for quantitative real-time PCR of mature sequences

\begin{tabular}{ll}
\hline miRNA/target & forward primer (5'-3') \\
\hline miR159a & GCGTTTGGATTGAAGGGAGCTCTA \\
miR159c & CGTTTGGATTGAAGGGAGCTCCT \\
miR164a & TGGAGAAGCAGGGCACGT \\
miR164b & TGGAGAAGCAGGGCACGT \\
miR166d-5p & AGGAATGTTGTCTGGCTCGAGG \\
miR396a & GCGCGTTCCACAGCTTTCTTGAACGT \\
miR396b-3p & CGGCTCAAGAAAGCTGTGGGAAA \\
miR396e-3p & CGCTCAAGAAAGCTGTGGGAGA \\
miR396g-3p & CGCTCAAGAAAGCCGTGGGAAAA \\
miR396h & GCGTCCACAGCTTTCTTGAACTG \\
miR408-3p & ATGCACTGCCTCTTCCCTGG \\
miR858a & GCGTTTCGTTGTCTGTTCGACCTT \\
novel_31 & CGCTTGGTTTGAAGGGAGCTCTA \\
novel_48 & GCTTCTATGTTCGAGGCCGAGT \\
novel_58 & GCGAATGGGAGTGCTGGTTAAACT \\
novel_70 & GGTGATGTTGGTTCGGTTCAGC \\
novel_164 & CGCGCGTAGATTCTTGAATTTGGCATA \\
novel_213 & CCCAGTGTTGCCCCCTCT \\
U6 & CCCTGCGCAAGGATGACACGC \\
\hline &
\end{tabular}

Table 2. Four pairs of primers for quantitative real-time PCR of target genes

\begin{tabular}{lll}
\hline target ID & forward primer $\left(5^{\prime}{ }^{\prime} 3^{\prime}\right)$ & reverse primer $\left(5^{\prime}{ }^{\prime}\right.$ ’’ $\left.^{\prime}\right)$ \\
\hline Gb_27117 & GGCTGGTATCGTGACAGATGAGATG & GCTCCTGCTGCCATTCCTGAAG \\
Gb_02586 & AGACCTCTGCTGCTGCTCCTAC & GCCTCCTGCGTAATTGCTGCTAG \\
Gb_00585 & ACTTCAATCGTGCTGCTGTATGTGG & GGAACTCGAATCTAGAAGAGGACTTGC \\
Gb_26816 & GCTTGCTCGTAAGGTTCATTCATCATG & GGTAATACCTCGCTGGTCCATATCTTG \\
GAPDH & CTGCCAAGGCTGTAGGTAAGG & TCAGATTCCTCCTTGATGGCG \\
\hline
\end{tabular}

adapter sequences, and other artifacts, we obtained 20,541,714 clean reads for further study.

The overall size distribution of filtered small RNAs from GBY was not even, with 21-nt small RNAs being the most abundant, representing $\sim 32.15 \%$ of the mutant leaf library (Fig. 2).

A total of 19,784,359 (96.31\%) reads from GBY were mapped to the genome database. The mapped

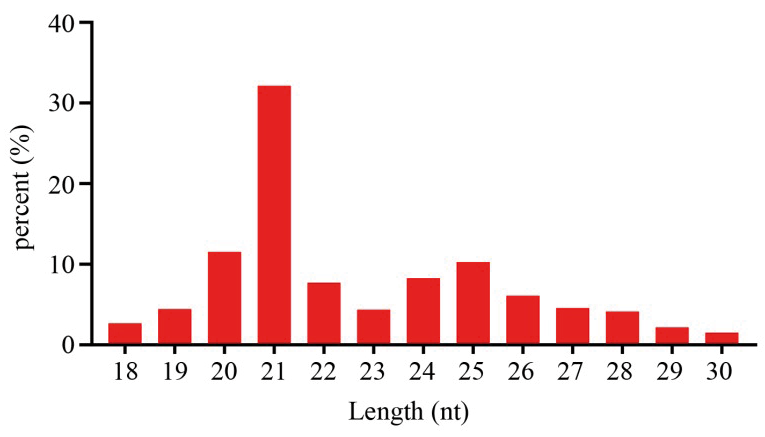

Figure 2. Length distribution of small RNAs from mutant leaves small RNA sequences were clustered into several RNA classes such as known miRNAs, rRNAs, tRNAs, snRNAs, snoRNAs, repeats, natural antisense transcripts, and trans-acting siRNAs. A total of 285,544 sequences were known miRNAs, representing $1.44 \%$ of GBY. Additionally, 1,985,687 sequences were identified as novel miRNAs in GBY.

\section{Identification of known and novel miRNAs}

The miRBase 21.0 was applied to annotate miRNAs of $G$. biloba based on our sequencing analysis. Ultimately, we identified 856 unique small RNAs for known miRNA analysis and 2,478 for novel miRNA analysis in GBY.

After screening, we obtained 213 mature known miRNAs matched to 54 miRNA families (Tab. 3). All precursors of mature miRNAs can adopt hairpin structures resembling the fold-back structure. Most known miRNA families have several members, 
Table 3. Summary of mapped mature and hairpin structures in known and novel miRNAs

\begin{tabular}{lcc}
\hline Types & Known & Novel \\
\hline Mapped total sRNA & 285544 & 1985687 \\
Mapped unique sRNA & 856 & 2478 \\
Mapped hairpin & 478 & 221 \\
Mapped mature & 213 & 214 \\
\hline
\end{tabular}

and the top five families (miR166, miR396, miR159, miR171, and miR167) have 25, 24, 22, 15, and 13 members, respectively (Fig. 3A). Thirtytwo miRNA families, almost half of the miRNA families identified, including miR157, miR394, and miR828, had only one family member.

We further analyzed the read counts in the known miRNA families and found great differences in the expression level among these miRNAs and miRNA families. miR166, miR159, and miR396 were the top three families in the two libraries. miR166 was the most abundant, with 398,443 reads in the constructed library (Fig. 2B). Additionally, miR162, miR167, miR319, and miR894 showed high expression levels in the library, with more than a thousand read counts, while several miRNAs (e.g., miR157, miR158, miR169, and miR403) were sequenced too scarce to be observed.

Novel miRNAs were also identified in the leaves. A characteristic stem-loop precursor is a prerequisite for annotating novel miRNAs. In total, 214 novel miRNA precursors were predicted from 5,222 unique small RNAs (Tab. 1). A total of 67 candidate novel miRNAs contained clear precursors with a stem-loop secondary structure. Among them, 36 novel miRNAs (e.g., novel_1, $12,189,15,14,17$, and 18) were highly expressed $(>10,000$ read counts) in the library. Novel_1 was represented the most frequently, with 1,016,266 copies in GBY (Fig. 3C). However, 35 novel

(A)

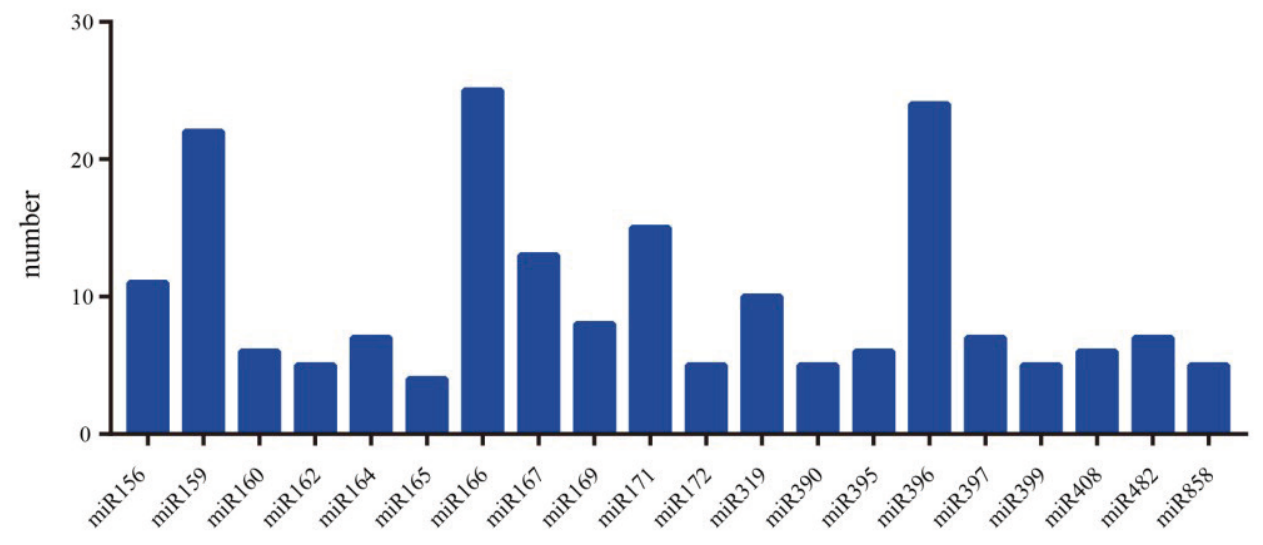

(B)

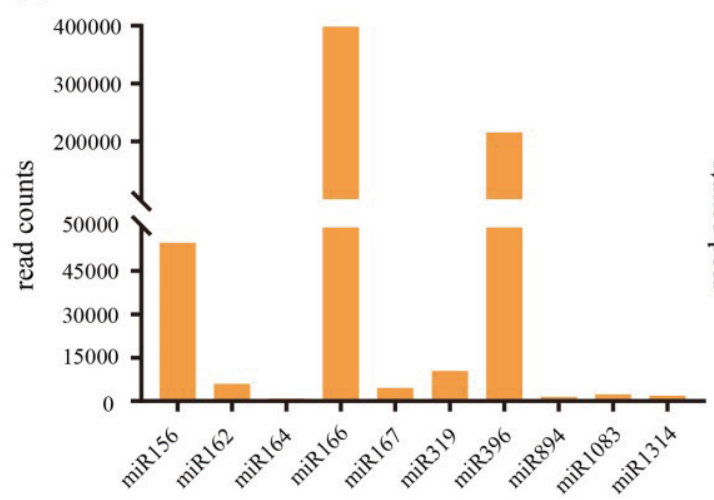

(C)

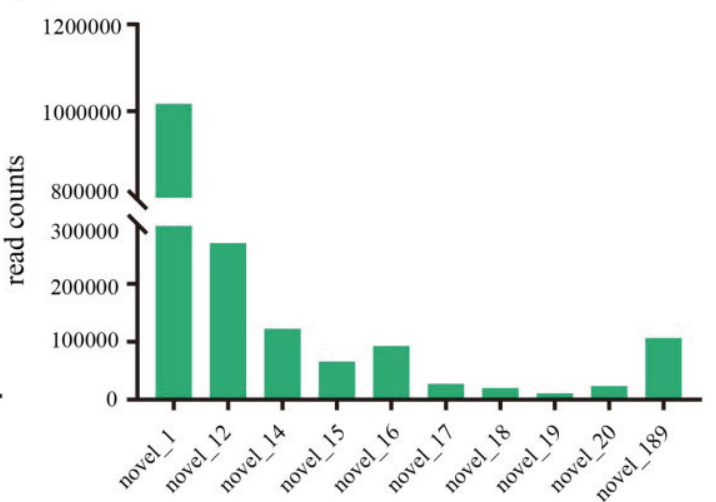

Figure 3. microRNAs (miRNAs) in mutant leaves: (A) numbers of members identified in the 20 conserved miRNA families; (B) read counts of the top 10 known miRNA families; (C) read counts of the top 10 novel miRNA families 
miRNAs (e.g., novel 166, 154, 173, 49, and 68) were lowly expressed $(<10$ read counts) in the library.

\section{Analysis of miRNAs and their targets in normal (green) and mutant leaves}

To explore the regulatory networks of miRNAs in the leaves of $G$. biloba, we constructed a library of small RNAs of the normal green leaf (GBG) as a control and compared the expression of miRNAs between GBG and GBY. After screening, 429 and 427 miRNAs were identified in GBG and GBY, respectively. To elucidate differentially expressed miRNAs between the leaves, we quantified the miRNAs in each sample and determined the miRNAs that differed significantly between the normal (green) and mutant leaves (fold-change $\mid \log _{2}$ (ratio) $\mid>1, p$-value $<0.05$ ). The results demonstrated that 42 known miRNAs and 54 novel candidate miRNAs showed different expression levels between GBG and GBY. Among them, 39 miRNAs were significantly up-regulated, and the remaining 57 miRNAs were down-regulated, in GBY.

Among the conserved miRNAs, 6 known miRNAs corresponding to two miRNA families, miR159 and miR396, had a higher expression abundance in GBY. In addition, more known miRNAs, i.e. 36 miRNAs corresponding to 17 miRNA families (e.g., miR164, miR166, and miR408) were significantly down-regulated in GBY. Interestingly, 2 miRNAs (miR397 and miR5077) were only expressed in GBG.

Among the non-conserved miRNAs, 33 novel miRNAs (e.g., novel_1, novel_14, novel_46, and novel_70) were preferentially expressed in GBY. Furthermore, 21 novel miRNAs (e.g., novel_21, novel 98, and novel 114) were found to be marginally represented in GBY. Notably, two novel candidate miRNAs (novel_287 and novel_344) were only expressed in GBY; meanwhile, four novel candidate miRNAs (novel_127, novel_136, novel_320, and novel_330) were only expressed in GBG.

Target genes of differentially expressed miRNAs were functionally annotated based on GO and KEGG analyses. The GO analysis showed that the putative target genes of known miRNAs were assigned to $1495 \mathrm{GO}$ terms. Among them, the most highly represented GO terms included 'DNA integration', followed by 'DNA metabolic process' and 'protein binding'. Moreover, biological interpretations of the target genes were further analyzed using the KEGG pathway database.
The results showed that a total of 58 biochemical pathways were involved with the target genes of the differentially expressed known and novel miRNAs. Among them, the most represented pathways included 'Glutathione metabolism', 'Plant circadian rhythm', and 'Phenylalanine metabolism', suggesting extensive miRNA function in gene regulatory networks.

\section{miRNA expression and target prediction}

In total, 2,867 miRNA-target pairs for known miRNAs and 1,766 for novel miRNAs were found. The number of predicted targets per miRNA varied from 1 to 29, and similar targets gathered around the same miRNA family. A total of 1,327 and 1,581 unigene sequences were predicted to be the targets of 244 known and 171 novel miRNAs, respectively. For example, miR164 was significantly downregulated in GBY. The potential targets of miR164 were implicated with a multitude of genetic functions such as cell expansion, cell division, shoot apical meristem (SAM) formation, leaf margin development, and leaf serration. miR397 targets the lac gene family, which is involved in lignin degradation. In addition, Gb_29451, which belongs to TO401 and is potentially targeted by miR396a, was directly involved in pore formation.

We particularly focused on miRNAs associated with chloroplast development according to their expression in the heat map and network. In the network of miRNAs and relative targets, we found 42 miRNA-target pairs (Fig. 4). Gb_27117, described as CLT3 and potentially regulated by several miR159 family members (miR159a, miR159a-3p, miR159c, and miR159f) (arrows, Fig. $4 \mathrm{~A}$ ), was involved in thiol transport from the plastid to the cytosol (Maughan and Buchanan, 2010). Several known miRNAs (miR396h, miR1511$3 \mathrm{p}$, miR5139, and miR8155) and novel miRNAs (novel_16, novel_164, novel_213, and novel_279) regulated similar potential targets (Gb_38129, $\mathrm{Gb}$ 26816, Gb_16854, Gb_04533, Gb_08053, $\mathrm{Gb}$ 33245, and $\mathrm{Gb}$ 30550) included in PPR32 (arrows, Fig. 4A), which was involved in multiple RNA editing events in chloroplasts (Okuda et al., 2009). miR408-3p, miR408b, and miR408d were highly down-regulated in GBY. Their target genes, HMA8 and RNR1 (arrows, Fig. 4A), were involved in copper transfer across the chloroplast thylakoid membrane and chloroplast division, respectively (Abdel-Ghany et al., 2005). 

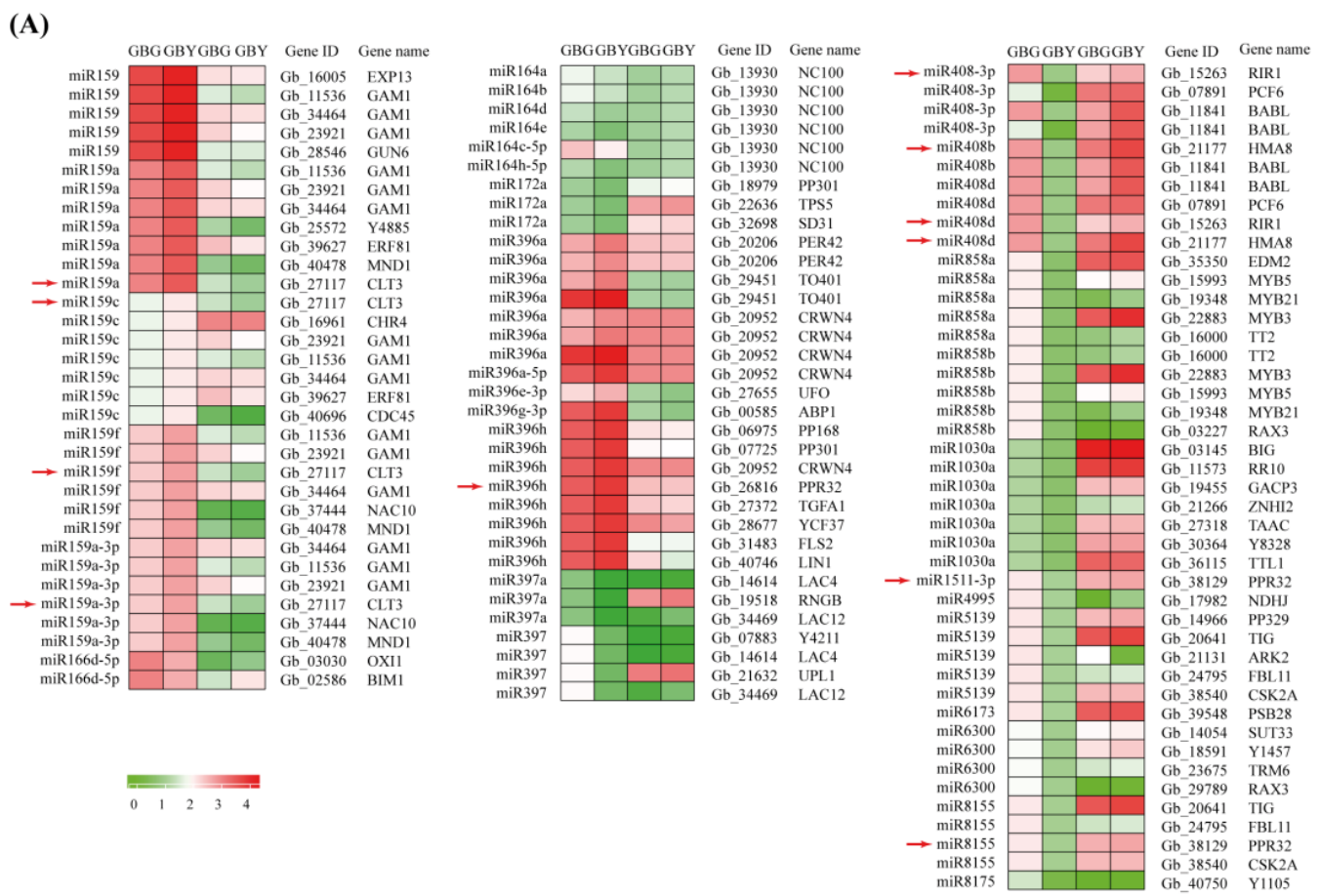

(B)
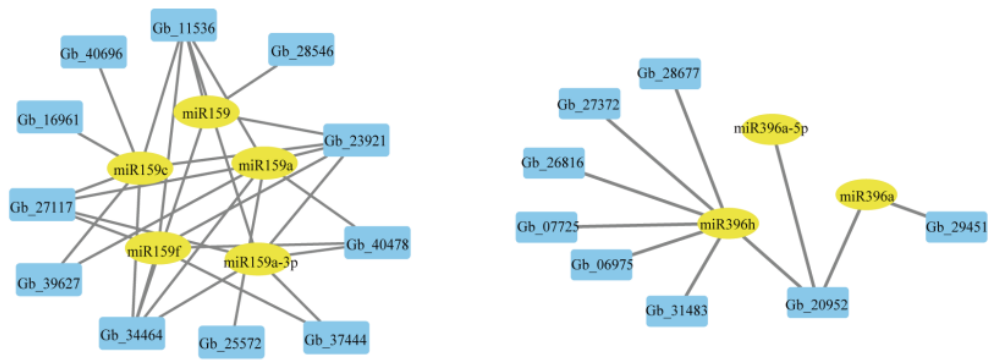

Figure 4. Heat maps and network of miRNAs and their targets. (A) A combined view of the inverse correlation between the expression of known miRNAs and target genes in GBG and GBY. The bar represents the expression scale for the miRNAs and target genes. Red indicates a high expression level, and green indicates a low expression level. The arrows point to miRNAs and their targets related to chloroplast development. (B) Network of miRNAs (yellow) and their targets (blue)

Auxins affect the entire progress of leaf development (Woodward and Bartel, 2005). Some miR396 family members (miR396a, miR396b$3 p$, miR396e-3p, and miR396g-3p) regulated several targets (Gb_17686, Gb_22331, Gb_11915, $\mathrm{Gb} 20206, \mathrm{~Gb} 1191 \overline{7})$ related to auxins. Gb ${ }^{-} 17686$, belonging to the FBX6 transcription factors, was involved in the regulation of the auxin-mediated signalling pathway, leaf morphogenesis, and leaf vascular tissue pattern formation. Gb_22331, Gb_11915, Gb_20206, and Gb_11917, described as PER42, were implicated in auxin catabolism. Gb_00585, which is potentially targeted by miR396 and was included in the ABP1 transcription factors, interacted functionally with phytochrome to regulate leaf development as an auxin receptor (Effendi et al., 2015).
The brassinosteroid (BR) class of steroid hormones regulates plant development and physiology. In particular, some BR proteins play important roles in leaf growth and stomatal and chloroplast development (Kim et al., 2012; Zhu et al., 2013). miR166 family members were downregulated in GBY. In the network of miRNAs and targets, we found that miR166d-5p targeted Gb_02586, which was described as BIM1 and is included in the positive brassinosteroid-signalling proteins (Yin et al., 2005).

\section{qRT-PCR validation of miRNAs and targets}

The expression levels of 12 known miRNAs and 6 novel miRNAs were analyzed. The relative transcript levels of 10 known miRNAs and 5 novel miRNAs had expression patterns similar 
(A)

qRT-PCR $\log _{2}(\mathrm{GBY} / \mathrm{GBG})$

deep sequencing $\log _{2}(\mathrm{GBY} / \mathrm{GBG})$

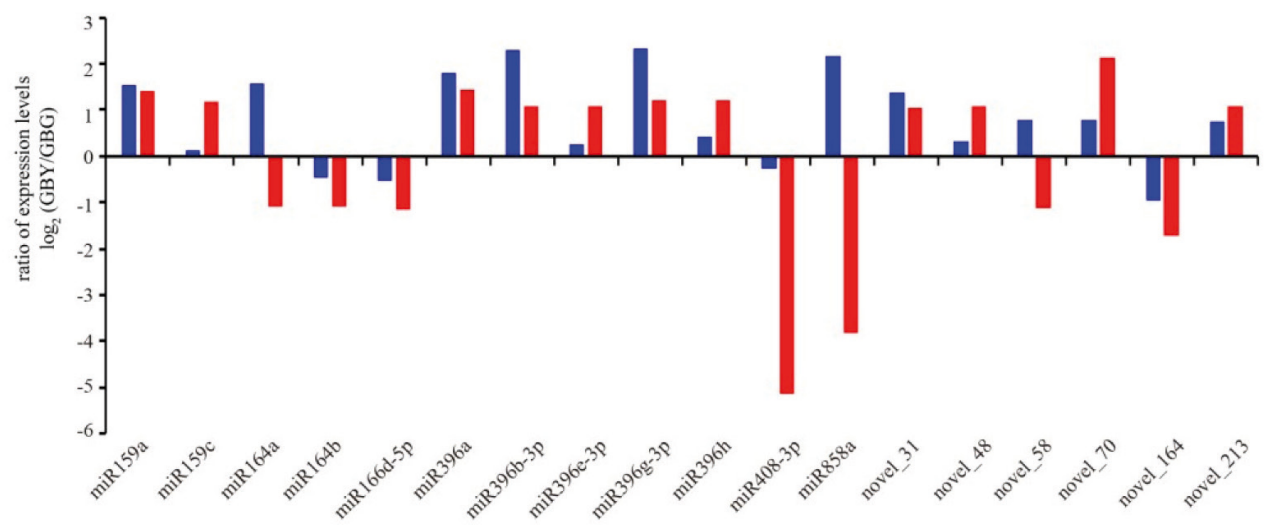

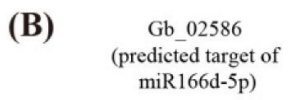

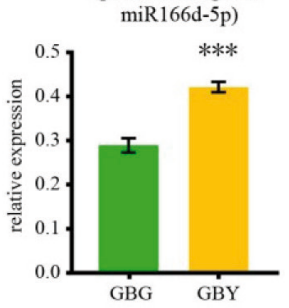

(C)

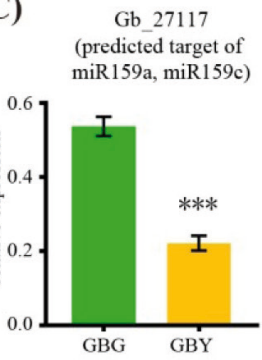

(D)

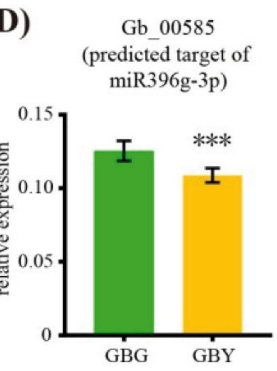

(E)
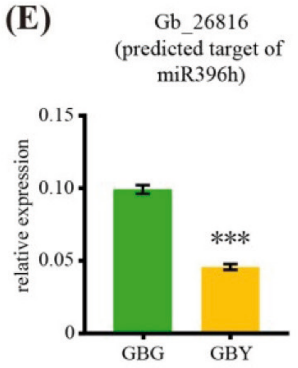

Figure 5. The expression ratios of miRNAs and their targets by qRT-PCR and deep sequencing: (A) the expression ratios $\left[\log _{2}(\mathrm{GBY} / \mathrm{GBG})\right]$ of miRNAs in G. biloba as determined by qRT-PCR and deep sequencing; (B-E) the bars indicate the expression levels of the corresponding miRNA targets, as determined by qRT-PCR. */*****: $p$-value $<0.05 / 0.01 / 0.001$, respectively

to those in the sequencing results, whereas two known miRNAs and one novel miRNA showed expression patterns that were opposite to those in the sequencing results (Fig. 5A).

Additionally, we analyzed the correlation between miRNAs and their identified targets. Five interesting miRNA/target modules were identified through the qRT-PCR analysis. A comparison of expression levels in the normal (green) leaves and mutant leaves revealed a negative relationship between miR159a and $\mathrm{miR} 159 \mathrm{c}$ and their corresponding targets. The expression level of Gb_27117 decreased in GBY. Conversely, that of the corresponding miR159a and miR159c increased (Fig. 5C). The expression level of Gb_02586 increased, while that of the corresponding miR166d-5p decreased (Fig. 5B). In addition, when the expression of miR396g$3 \mathrm{p}$ and miR396h dramatically increased in GBY, their respective targets, Gb_00585 and Gb_26816, inversely decreased (Fig. 5D, 5E).

\section{DISCUSSION}

Complex networks of gene regulatory pathways participate in leaf development in plants. miRNAs are known key components of the networks and play a variety of important regulatory roles. These miRNAs are perfectly or imperfectly complementary to their targeted $m R N A s$ and repress the target genes by mRNA cleavage or translation suppression. Notably, plant miRNAs and their target genes are conserved, which allows fast and confident bioinformatic identification of the targets (Rubiosomoza and Weigel, 2011). Previously, we analyzed the miRNAs in mature leaves of G. biloba and found 202 and 201 known miRNAs belonging to 82 and 78 families in female and male leaves, respectively (Wang et al., 2015). In addition, in the ovules of female trees and the ovules of G. biloba var. epiphylla, 34 and 82 conserved miRNAs, respectively, were also identified (Wang et al., 2016; Zhang et al., 2015). In the current study, we identified miRNAs and their targets in a leaf colour mutant of G. biloba, and obtained 213 potentially known miRNAs belonging to 54 miRNA families in mutant leaves that were different from those in the mature normal leaves of G. biloba, and the number was significantly higher than the number of ovules, indicating a diverse array of miRNAs 
involved in colour formation in the G. biloba leaf mutant.

A large majority of plant small RNAs are 21 or $24 \mathrm{nt}$ in length. Many angiosperms have a major peak at $24 \mathrm{nt}$, while the length distribution patterns of small RNAs from gymnosperms, including Tasus chinensis, Pinus contorta, and seven other conifer species, show a major peak at $21 \mathrm{nt}$, with no obvious peak at $24 \mathrm{nt}$ (Ding et al., 2014; Dolgosheina et al., 2008; Wei et al., 2011). Moreover, the major peak or proportion of miRNAs varies among plant species and organs (Wan et al., 2012). For example, the mature leaves of G. biloba only displayed a 21nt major peak, whereas there was a major peak at $21 \mathrm{nt}$ and a minor peak at $24 \mathrm{nt}$ in the ovules of G. biloba. In the present study, the small RNA length distribution for GBY showed a major peak at $21 \mathrm{nt}$ and no obvious peak at $24 \mathrm{nt}$, similar to other gymnosperms and the mature leaves of G. biloba, suggesting that small RNA length distribution patterns are highly conserved in the same organs, but differ considerably among different organs.

The regulation mechanism of leaf colour formation is varied and complex. The main factors are the effects of chloroplast synthesis and the expression of proteins in chloroplast transcription. Previous studies had reported that the chloroplast structure was different between mutants and normal green leaves (Yoo et al., 2009). In bamboo albino leaves, thylakoid membranes were converted into numerous abnormal vesicles in the chloroplast, compared to those in normal green leaves (Yang et al., 2015a). Similarly, in Anthurium andraeanum mutant leaves, the chloroplasts accumulated inflated starch granules leading to the formation of big gaps between stroma thylakoids, whereas in normal leaves the chloroplasts only contained small starch granules (Yang et al., 2015b). In our previous study of G. biloba mutant leaves, the chloroplast structure in mutants was significantly different from that of normal leaves. The mutant leaves displayed fewer and smaller chloroplasts and exhibited an altered ultrastructure, indicating an abnormal development of chloroplasts in the colour-mutated leaves of $G$. biloba.

In this study, by further identifying the differential expression of miRNAs between GBG and GBY, we also found direct development-related miRNAs involved in chloroplast development. PPR32 is reportedly involved in multiple RNA editing events in chloroplasts. In GBY, PPR32 transcription factors were predicted to be targeted by several known miRNAs (miR396h, miR1511- 3p, miR5139, and miR8155) and novel miRNAs (novel 164 and novel 213). The expression levels of these miRNAs were very significantly increased in GBY compared to GBG. The qRT-PCR results further confirmed the importance of miR396h and the predicted target PPR32 in GBY. In addition, several other miRNAs that are related to chloroplast development and chlorophyll metabolism have been identified as playing important roles in leaf development, such as miR156, miR159, miR166, and miR319 (Mallory et al., 2004; Millar and Gubler, 2005; Ori et al., 2007; Xie et al., 2012). For example, in Lemna gibba, an endogenous miR166 precursor was used as a backbone to produce artificial miRNAs, whose expression could result in reduced frond pigmentation and a significant decrease of approximately $40 \%$ in chlorophyll content (Cantópastor et al., 2015). Moreover, in Arabidopsis, miR171c overexpressors and scl6 scl22 scl27 triple mutants produce dark green leaves, which contain approximately $40 \%$ more chlorophyll than wild-type leaves do. In contrast, the overexpression of miR171-resistant $L U C$ rSCL27 results in leaf yellowing and a significant decrease in chlorophyll content, indicating that miR171-targeted SCLs are negative regulators of chlorophyll biosynthesis (Wang et al., 2010). Here, we found that the expression of miR159a and miR159c was significantly up-regulated in GBY. Through the web-based software psRNATarget and psRobot_tar in psRobot, we identified their target as Gb_27117, described as CLT3, which is involved in thiol transport in plastids. Combing the ultrastructure differences of chloroplasts in the colour mutant of G. biloba, we hypothesized that miR159 and miR396 might participate in regulating leaf colour formation by regulating chloroplast development and chlorophyll biosynthesis.

Phytohormones control almost all developmental stages across the plant life cycle, and miRNAs, as key regulators, affect their metabolism, distribution, and perception. A number of studies have shown that phytohormones such as cytokinins, auxins, and brassinosteroids are also involved in regulating the development and activity of chloroplasts (Cortleven and Schmülling, 2015; Komatsu et al., 2010). In Arabidopsis, miR160 and miR167 have been implicated in the down-regulation of several classes of ARF genes, and miR393 targets auxin receptors of the TIR1/AFB family. miR390 also controls the expression of several ARFs, but using a more complicated mechanism (Bologna and Voinnet, 2014; Martínez de Alba et al., 2013; 
Rhoades et al., 2002). In Physcomitrella patens, miR1221 was predicted to target the AHK4 cytokinin receptor (Talmor-Neiman et al., 2006), and miR1027 showed possible complementarity to the cytokinin oxidase gene CKX1 (Axtell et al., 2007). In this study, we predicted that miR166d-5p targeted the brassinosteroid-related gene Gb_02586. Some miR396 family members, such as miR396a, miR396b-3p, miR396e-3p, and miR396g-3p, regulated several targets (Gb_00585, Gb_17686, $\mathrm{Gb}$ 22331, Gb_11915, Gb_20206, and Gb_11917) associated with auxins. Further qRT-PCR analysis revealed negative relationships between miR166d$5 \mathrm{p}, \mathrm{miR} 396 \mathrm{~g}-3 \mathrm{p}$ and their corresponding targets (Gb_02586, Gb_00585). It is likely that miR166d$5 \mathrm{p}$ and miR396g-3p affect hormone metabolismrelated genes involved in leaf coloration.

\section{CONCLUSIONS}

We constructed libraries of small RNAs of goldengreen striped mutant leaves and normal leaves of G. biloba. We identified 42 known miRNAs and 54 novel differentially expressed candidatemiRNAs; 39 were up-regulated and 57 down-regulated in GBG and GBY. Furthermore, by combining these findings with annotations of the predicted targets and the results of qRT-PCR analysis, we found that the differentially expressed miRNA target genes were involved in chloroplast development and hormone metabolism. For example, miR159a, miR159a-3p, miR159c, and miR159f targeted CLT3; miR396h, miR1511-3p, miR5139, miR8155, and novel_16, 164, 213, and 279 regulated similar potential targets included in PPR32, which is associated with chloroplast development. In addition, miR396h and miR396g-3p were dramatically increased in GBY, while miR166 family members were downregulated; the target genes of both were involved in hormone metabolism. Given that our previous transcriptional sequence analysis had revealed that changes in the expression of genes related to chloroplast development, chlorophyll biosynthesis, and degradation resulted in a leaf colour mutant, we suggest that some miRNAs, such as miR159, miR166, and miR396, together with their target genes, potentially play important roles in leaf colour mutation by regulating chloroplast development and hormone metabolism.

\section{ACKNOWLEDGEMENT}

We thank Novogene Company (Beijing) for helping with sequencing and technical assistance.

\section{FUNDING}

This work was supported financially by Natural Science Foundation of China (No. 31670181; No. 31670695), National Key R \& D Program of China (No. 2017YFD0600701), Graduate student scientific research innovation projects in Yangzhou university (No. XKYCX18_089).

\section{AUTHOR CONTRIBUTIONS}

W.L., Z.H. and L.W. - conceived and designed the experiments; Z.H., S.Y. and Y.Y. - performed the experiments; Z.H., Y.Y. and H.J. - analyzed the data; W.L., Z.H. and L.W. - wrote the paper.

\section{CONFLICT OF INTEREST}

Authors declare no conflict of interest.

\section{REFERENCES}

Abdel-Ghany S.E., Müller-MouléP., NiYogi K.K., Pilon M., Shikanai T., 2005. Two P-Type ATPases are required for copper delivery in Arabidopsis thaliana chloroplasts. Plant Cell 17(4), 1233-1251.

Adhikari N.D., Froehlich J.E., Strand D.D., Buck S.M., Kramer D.M., Larkin R.M., 2011. GUN4-porphyrin complexes bind the CNH/GUN5 subunit of Mg-Chelatase and promote chlorophyll biosynthesis in Arabidopsis. Plant Cell 23(4), 1449.

ARChETti M., 2000. The origin of autumn colours by coevolution. J. Theor. Biol. 205, 625-630.

Axtell M.J., SNyder J.A. BArTel D.P., 2007. Common functions for diverse small RNAs of land plants. Plant Cell 19(6), 1750-1769.

Bologna N.G., VoInnet O., 2014. The diversity, biogenesis, and activities of endogenous silencing small RNAs in Arabidopsis. Annu. Rev. Plant Biol. 65(1), 473-503.

Cantópastor A., Mollámorales A., Ernst E., Dahl W., Zhai J., YAN Y., et AL., 2015. Efficient transformation and artificial miRNA gene silencing in Lemna minor. Plant Biol. 17(s1), 59-65.

Cortleven A., Schmülling T., 2015. Regulation of chloroplast development and function by cytokinin. J. Exp. Bot. 66(16), 4999.

Ding Q., Zeng J., He X.Q., 2014. Deep sequencing on a genome-wide scale reveals diverse stage-specific microRNAs in cambium during dormancy-release induced by chilling in poplar. BMC Plant Biol. 14(1), 267.

Dolgosheina E.V., Morin R.D., Aksay G., Sahinalp S.C., Magrini V., Mardis E.R., et Al., 2008. Conifers have a unique small RNA silencing signature. RNA-a Publication of the RNA Society 14(8), 1508-1515.

Effendi Y., Ferro N., Labusch C., Geisler M., Scherer G.F.E., 2015. Complementation of the 
embryo-lethal T-DNA insertion mutant of $A U X I N$ BINDING-PROTEIN 1 (ABP1) with abpl point mutated versions reveals crosstalk of ABP1 and phytochromes. J. Exp. Bot. 66(1), 403-418.

Eriksson M., Moseley J.L., Tottey S., Del Campo J.A., Quinn J., Kim Y., ET AL., 2004. Genetic Dissection of Nutritional Copper Signaling in Chlamydomonas Distinguishes Regulatory and Target Genes. Genetics 168(2), 795.

Garton S., Knight H., Warren G., Knight M.G., 2007. Crinkled leaves 8 - A mutation in the large subunit of ribonucleotide reductase - leads to defects in leaf development and chloroplast division in Arabidopsis thaliana. Plant J. 50(1), 118-127.

Guan X., Pang M., Nah G., Shi X., Ye W., Stelly D.M., ET AL., 2014. miR828 and miR858 regulate homoeologous MYB2 gene functions in Arabidopsis trichome and cotton fibre development. Nat. Commun. 5(1), 3050.

Horie Y., Ito H., Kusaba M., Tanaka R., Tanaka A., 2009. Participation of chlorophyll $b$ reductase in the initial step of the degradation of light-harvesting chlorophyll a/b-protein complexes in Arabidopsis. J. Biol. Chem. 284(26), 17449-17456.

Kim T.W., Michniewicz M., Bergmann D.C., Wang Z.Y., 2012. Brassinosteroid regulates stomatal development by GSK3-mediated inhibition of a MAPK pathway. Nature 482(7385), 419.

Komatsu T., Kawaide H., Saito C., Yamagami A., Shimada S., NaKazawa M., et al., 2010. The chloroplast protein BPG2 functions in brassinosteroid-mediated post-transcriptional accumulation of chloroplast rRNA. Plant J. 61(3), 409-422.

Lee D.W., O’Keefe J., Holbrook N.M., Feild T.S., 2003. Pigment dynamics and autumn leaf senescence in a new england deciduous forest, eastern USA. Ecol. Res. 18, 677-694.

Li W.X., Yang S.B., Lu Z.G., He Z.C., Ye Y.Y., ZhaO B.B., ET AL., 2018. Cytological, physiological, and transcriptomic analyses of golden leaf coloration in Ginkgo biloba L. Hortic. Res. 5(1), 12.

Li Y.H., WANG B.H., DAi Z.Y., Li A.H., LiU G.Q., ZuO S.M., ET AL., 2012. Morphological structure and genetic mapping of new leaf-color mutant gene in rice (Oryza sativa). Rice Sci. 19(2), 79-85.

Livak K.J., Schmittgen T.D., 2001. Analysis of relative gene expression data using real-time quantitative PCR and the $2^{-\Delta \Delta \mathrm{ct}}$ method. Methods 25,402-408.

Lonosky P.M., Zhang X., Honavar V.G., Dobbs D.L., Fu A., Rodermel S.R., 2004. A proteomic analysis of maize chloroplast biogenesis. Plant Physiol. 134(2), 560-574.

Maekawa S., Takabayashi A., Huarancca R.T., Yamamoto H., Tanaka A., Sato T., et Al., 2015. Pale-green phenotype of atl31 atl6 double mutant leaves is caused by disruption of 5-aminolevulinic acid biosynthesis in Arabidopsis thaliana. PLOS ONE 10(2), e0117662.

Mallory A., Reinhart B.R., Matthew J.R., TAng G., Zamore P., BARTON M., ET AL., 2004. MicroRNA control of PHABULOSA in leaf development: importance of pairing to the microRNA 5' region. Embo J. 23(16), 3356-3364.

Maple J., MÃLler S.G., 2007. Plastid division: evolution, mechanism and complexity. Ann. Bot 99(4), 565-579.

Martínez de Alba A.E., Elvira-Matelot E., Vaucheret H., 2013. Gene silencing in plants: a diversity of pathways. Biochim. Biophys. Acta, Gene Regul. Mech. 1829(12), 1300-1308.

Maughan S.C., Buchanan B.B., 2010. Plant homologs of the Plasmodium falciparum chloroquineresistance transporter, PfCRT, are required for glutathione homeostasis and stress responses. Proc. Natl. Acad. Sci. USA. 107(5), 2331-2336.

Millar A.A., Gubler F., 2005. The Arabidopsis GAMYB-like genes, MYB33 and MYB65, are microRNA-regulated genes that redundantly facilitate anther development. Plant Cell 17(3), 705721.

Mochizuki N., Brusslan J.A., Larkin R., Nagatani A., CHORY J., 2001. Arabidopsis genomes uncoupled 5 (GUN5) mutant reveals the involvement of $\mathrm{Mg}$ chelatase $\mathrm{H}$ subunit in plastid-to-nucleus signal transduction. Proc. Natl. Acad. Sci. USA. 98(4), 2053-2058.

Moon J., Zhu L., Shen H., HuQ E., 2008. PIF1 directly and indirectly regulates chlorophyll biosynthesis to optimize the greening process in Arabidopsis. Proc. Natl. Acad. Sci. USA. 105(27), 9433-9438.

Nagata N., Tanaka R., Satoh S., TanaKa A., 2005. Identification of a vinyl reductase gene for chlorophyll synthesis in Arabidopsis thaliana and implications for the evolution of Prochlorococcus species. Plant Cell 17(1), 233.

Okuda K., Chateigner-Boutin A.L., NaKamura T., Delannoy E., Sugita M., Myouga F., ET Al., 2009. Pentatricopeptide repeat proteins with the DYW motif have distinct molecular functions in RNA editing and RNA cleavage in Arabidopsis chloroplasts. Plant Cell 21(1), 146.

Ori N., Cohen A.R., Etzioni A., Brand A., Yanai O., Shleizer S., et AL., 2007. Regulation of LANCEOLATE by miR319 is required for compound-leaf development in tomato. Nat. Genet. 39(6), 787-791.

Pan Y., Bradley G., Pyke K., Ball G., Lu C., Fray R., ET AL., 2013. Network inference analysis identifies an APRR2-like gene linked to pigment accumulation in tomato and pepper fruits. Plant Physiol. 161(3), 1476-1485.

Papenbrock J., Mock H.P., Tanaka R., Kruse E., Grimm B., 2000. Role of magnesium chelatase activity in the early steps of the tetrapyrrole 
biosynthetic pathway. Plant Physiol. 122(4), 11611169.

Rhoades M.W., Reinhart B.J., Lim L.P., Burge C.B., Bartel B., Bartel D.P., 2002. Prediction of plant microRNA targets. Cell 110(4), 513-520.

Rubiosomoza I., Weigel D., 2011. MicroRNA networks and developmental plasticity in plants. Trends Plant Sci. 16(5), 258-264.

Sato Y., Morita R., Nishimura M., Yamaguchi H., Kusaba M., 2007. Mendel's green cotyledon gene encodes a positive regulator of the chlorophylldegrading pathway. Proc. Natl. Acad. Sci. USA. 104(35), 14169-14174.

Talmor-Neiman M., Stav R., Frank W., Voss B., Arazi T., 2006. Novel micro-RNAs and intermediates of micro-RNA biogenesis from moss. Plant J. 47(1), $25-$ 37.

Wan L.C., Feng W., Guo X., Lu S., Qiu Z., Zhao Y., ET AL., 2012. Identification and characterization of small non-coding RNAs from Chinese fir by high throughput sequencing. BMC Plant Biol. 12(1), 146.

Wang L., Lu Z., Li W., Xu J., Luo K., Lu W., ET AL., 2016. Global comparative analysis of expressed genes in ovules and leaves of Ginkgo biloba L. Tree Genet. Genomes 12(2), 29.

Wang L., MaI Y.X., Zhang Y.C., Qian L., Yang H.Q., 2010. MicroRNA171c-targeted SCL6-II, SCL6-III, and SCL6-IV genes regulate shoot branching in Arabidopsis. Mol. Plant 3(5), 794-806.

Wang L., Zhao J., Zhang M., Li W., Luo K., Lu Z., ET AL., 2015. Identification and characterization of microRNA expression in Ginkgo biloba L. leaves. Tree Genet. Genomes 11(4), 76.

Wei L.Q., Yan L.F., WANG T., 2011. Deep sequencing on genome-wide scale reveals the unique composition and expression patterns of microRNAs in developing pollen of Oryza sativa. Genome Biol. 12(6), R53.

Woodward A.W., BArtel B., 2005. Auxin: regulation, action, and interaction. Ann. Bot. 95(5), 707-735.
Wu Z., Zhang X., He B., Diao L., Sheng S., Wang J., ET AL., 2007. A chlorophyll-deficient rice mutant with impaired chlorophyllide esterification in chlorophyll biosynthesis. Plant Physiol. 145(1), 29.

Xie K., Shen J., Hou X., Yao J., Li X., Xiao J., et AL., 2012. Gradual increase of miR156 regulates temporal expression changes of numerous genes during leaf development in rice. Plant Physiol. 158(3), 1382-1394.

Yang H.Y., Xia X.W., Fang W., Fu Y., An M.M., ZHou M.B., 2015a. Identification of genes involved in spontaneous leaf color variation in Pseudosasa japonica. Genet. Mol. Res. 14(4), 11827-11840.

Yang Y., Chen X., Xu B., Li Y., Ma Y., Wang G., 2015b. Phenotype and transcriptome analysis reveals chloroplast development and pigment biosynthesis together influenced the leaf color formation in mutants of Anthurium andraeanum 'Sonate'. Front Plant Sci. 6, 139.

Yin Y., Vafeados D., Tao Y., Yoshida S., Asami T., CHORY J., 2005. A new class of transcription factors mediates brassinosteroid-regulated gene expression in Arabidopsis. Cell 120(2), 249-259.

Yoo S.C., Cho S.H., Sugimoto H., Li J.J., Kusumi K., КоH H.J., ET AL., 2009. Rice virescent3 and stripel encoding the large and small subunits of ribonucleotide reductase are required for chloroplast biogenesis during early leaf development. Plant Physiol. 150(1), 388-401.

Zhang Q., Li J., SAng Y., XIng S., Wu Q., LiU X., 2015. Identification and characterization of MicroRNAs in Ginkgo biloba var. epiphylla Mak. PLOS ONE 10(5), e0127184.

Zhu J.Y., SAeSEAW J., WANG Z.Y., 2013. Brassinosteroid signalling. Development 140(8), 1615-1620.

Received August 2, 2018; accepted November 20, 2018 